Individual Psychotherapy and the Science of Psychodynamics

By David H. Malan. Pp. xii + 275. Butterworths, London, Boston, Sydney, Wellington, Durban and Toronto, 1979. $£ 8.00$.

Medical psychotherapists usually enrage their clinical and academic colleagues by their bland and sometimes seemingly defensive refusal to conduct conventional 'outcome' research. Dr Malan is an exception. Morevoer, though a psychoanalist at heart, he has retained the capacity to relate the uniqueness of individual experience to the generalities of social life. Measurements in the latter sphere can genuinely reflect the former and changes in the former. Probably as a consequence he is able to think more clearly, and in a demystifying way, about the practical issues of psychotherapy. Does it work and under what circumstances does it work and what light can its impact throw on our further understanding of human experience? The present book is a tribute to him. It is simply and clearly written. Medical and nonmedical readers, givers and receivers of psychotherapy can all benefit from reading it. Successive sections of the book deal with such matters, all illustrated by attractively presented case material, as the nature of some psychological mechanisms; their relevance to human relationships, especially with would-be therapists; gender identity; the potentially healing experience of depression and the nature of the task of assessment for treatment. This is not a book primarily concerned with research method. Clinicians however are likely to find themselves time and time again identifying with the many truths within it. It is a profoundly clinical book and one of the best of its kind.

\section{Mathematics for Biomedical Applications}

By Stanton A. Glantz. Pp. 423. University of California Press. London, 1979. £17.75.

The scope of this book is somewhat more restricted than its title suggests. It contains, for example, virtually no mention of statistical techniques. The author concentrates exclusively on the mathematics of dynamic processes, i.e. differential equations (excluding partial differential equations).

After describing the formulation of differential equations he discusses direct and indirect methods of solution, and the use of computers, indicating the possible inaccuracies which may arise using numerical methods. He includes a chapter on Fourier analysis, with special reference to the recording of cardiovascular pressure pulses. Appendices contain a review of calculus, a table of commonly used integrals, an introduction to the use of complex numbers and functions, and a table of Laplace transform pairs. Each chapter has problems relating to its subject matter, and an appendix gives the solution. There is a good index. Moderate familiarity with mathematical symbols and notation is assumed.

I doubt whether this book in itself would provide a reader unfamiliar with the calculus with sufficient basis for more than a nodding acquaintance with the application of linear systems theory to biological problems. For someone who has some familiarity with the calculus it is well designed to help him understand and manipulate the fundamental tools used in the field, although there are many gaps, and some material given in tables of formulae is not explained or derived.

The examples chosen are mainly from the fields of pharmacokinetic, cardiological and circulatory research with which the author is familiar. The worked examples are very well explained and imaginatively illustrated, pictorially. That is, indeed, one of the main strengths of the book and will greatly help those readers who are less mathematically literate. On the debit side there are a number of arithmetical or notational errors in the worked examples, e.g. equations $2.76,2.77,3.223,3.265,4.46$. Limitations of analyses are usually only briefly considered. The sections on indicator dilution and transducer response and filtering are particularly good.

In general the book can be highly recommended.

\section{Laboratory Investigation of Endocrine Disorders}

By M. R. Wills, C. W. H. Harvard and P. J. Roylance. Pp. 96. Butterworths, London, 1979. $£ 3.25$.

This is a most helpful little book which is a valuable guide to the houseman and research fellow in an endocrine unit and those where metabolic investigations are being undertaken. Current practice is that these are undertaken on an outpatient basis and the information in this little book is very helpful. Practical details and safeguards are very adequately noted in the text which is very clearly set out and easy to read. The basic principles of all the investigations are outlined but such explanations might have been improved by simple diagrams although the tables are quite helpful.

It may also have been helpful to insert just a few clinical details which would lead one to undertake certain tests and it is perhaps a pity that hypoglycaemia is not included either as an entity to be investigated or as a complication of some of the disorders to be discussed.

This book would have benefited by having an index as well as a contents list and if perhaps less money were to have been spent on the rather glossy but attractive cover it would have brought down the cost which even in these days of expensive printing seems a little high.

All in all, it is a very valuable little handbook useful to those who may spend time looking through the larger endocrine tests to delineate the most appropriate investigations. The anthors are to be congratulated on its production.

\section{Medical Immunology}

Edited by James Irvine. Pp. ix +506 , illustrated. Teviot Scientific Publications, Edinburgh, 1979. $£ 12.50$ (paperback) f16.00 (hardback).

Immunology has infiltrated every branch of clinical medicine. Nevertheless, its impact on the actual treatment of patients has been disappointing; so far, few potent therapeutic tools have emerged. The laboratory techniques developed have enjoyed a wide application and our understanding of the pathogenic mechanisms operating in various diseases have increased dramatically. There can be few disorders which have not been submitted to the scrutiny of, at least, antibody screening, T lymphocyte function tests and HLA typing. The crucial question regarding the primary or secondary nature of these phenomena is a conundrum which confuses most clinicians. This book certainly puts these observations into perspective, even if it does not provide many answers. It assumes a knowledge of the standard medical texts and is aimed both at the general physician and the basic scientist wishing to learn more of the clinical relevance of his work. Three introductory chapters covering basic immunology and tissue typing lead into sections on the main systems of the body, immunodeficiency, transplantation and tumour immunology. Paediatrics is not specifically covered. A multiauthor format is used, the approach being based on the annual Edinburgh course on clinical immunology. Bibliographies of varying lengths are provided at the end of each chapter and there is an extensive index.

The system chapters describe the immunological features associated with the relevant disorders and discuss the mechanisms of tissue damage and the implications for diagnosis and treatment. There is, inevitably, some repetition and there is some inconsistency in the use of terminology; however, the overall balance of the book is excellent and the style is eminently readable. This derives in part from 